\title{
BMJ Open Community-based physical activity interventions among women: a systematic review
}

\author{
Leila Amiri Farahani, ${ }^{1}$ Mohsen Asadi-Lari, ${ }^{2}$ Eesa Mohammadi, ${ }^{3}$ Soroor Parvizy,,${ }^{4,5}$ \\ Ali Akbar Haghdoost, ${ }^{6}$ Ziba Taghizadeh $^{7}$
}

To cite: Amiri Farahani L, Asadi-Lari M, Mohammadi E, et al. Community-based physical activity interventions among women: a systematic review. BMJ Open 2015;5: e007210. doi:10.1136/ bmjopen-2014-007210

- Prepublication history for this paper is available online. To view these files please visit the journal online (http://dx.doi.org/10.1136/ bmjopen-2014-007210).

Received 28 November 2014 Revised 2 March 2015 Accepted 3 March 2015
CrossMark

For numbered affiliations see end of article.

Correspondence to Dr Ziba Taghizadeh; taghizad@tums.sina.ac.ir

\section{ABSTRACT}

Objective: Review and assess the effectiveness of community-based physical activity interventions among women aged 18-65 years.

Design: Systematic review

Methods: To find relevant articles, the researcher selected reports published in English between 1 January 2000 and 31 March 2013. Systematic search was to find controlled-trial studies that were conducted to uncover the effect of community-based interventions to promote physical activity among women 18-65 years of age, in which physical activity was reported as one of the measured outcomes. The methodological quality assessment was performed using a critical appraisal sheet. Also, the levels of evidence were assessed for the types of interventions.

Results: The literature search identified nine articles. Four of the studies were randomised and the others studies had high methodological quality. There was no evidence, on the basis of effectiveness, for social cognitive theory-based interventions and inconclusive evidence of effectiveness for the rest of interventions.

Conclusions: There is insufficient evidence to assess the effectiveness of community-based interventions for enhancing physical activity among women. There is a need for high-quality randomised clinical trials with adequate statistical power to determine whether multicomponent and community-based intervention programmes increase physical activity among women, as well as to determine what type of interventions have a more effective and sustainable impact on women's physical activity.

\section{INTRODUCTION}

Physical activity (PA) is recognised as one of the most important behaviours for reducing the overall burden of disease in humans. ${ }^{1}$ The leading causes of death worldwide are primarily found among four noncommunicable diseases (NCDs): cardiovascular diseases, cancers, diabetes and chronic respiratory diseases. The burdens of these diseases are considerably heavier in developing and low-income countries where the rates of these NCDs continue to climb. ${ }^{2}$ Developing

\section{Strengths and limitations of this study}

This is the first review to explore the effectiveness of community-based physical activity interventions among women aged 18-65 years.

- The trial screening and data extraction were conducted using the strong appraisal sheets, independently by two authors.

- Owing to heterogeneity in the types of community-based interventions, methodology quality and the impossibility of searching all electronic and non-electronic databases with a language restriction, the ability of achieving strong (solid) conclusions might be limited.

countries have been experiencing a rapid phase of unplanned urbanisation and industrialisation, population-ageing and globalisation. These result in unhealthy environments, with rapid social and economic transition accompanied by changes in PA. As a result, the growing prevalence of NCDs and their risk factors has become a global issue in undeveloped and developing countries. ${ }^{3}$

By 2030, low-income countries will have eight times more deaths attributed to NCDs than high-income countries. ${ }^{2}$ The WHO estimates that $80 \%$ of all deaths may be attributed to NCDs by $2020 .^{4}$

Tobacco use, harmful use of alcohol, insufficient PA and unhealthy diet are the four main behavioural risk factors which induce NCDs and are expected to rise in developing countries. ${ }^{5}$

In reference to the US physical activity guideline (2008), there is strong evidence that $\mathrm{PA}$ reduces the risk of many adverse health outcomes, such as early death, coronary heart disease, stroke, high-blood pressure, adverse blood lipid profile, type 2 diabetes, metabolic syndrome and depression; ${ }^{1}$ also PA is considered an independent cancer-protective factor. ${ }^{6}$

Although there are many benefits in adopting PA, its rates have remained low. ${ }^{7}$ Dumith 
et $a l^{8}$ have presented a comprehensive worldwide estimation of physical inactivity in which the overall prevalence of physical inactivity was $21.4 \%$, that is, $23.7 \%$ prevalence for women and $18.9 \%$ prevalence for men; however, their report was limited by many factors, such as not having access to data from many populous countries, and using a self-report questionnaire that caused underestimation of physical inactivity.

According to the second report of the urban health equity assessment and response tool (Urban HEART) project conducted in 2011 in Tehran, Iran, only 20.5\% women and $24.3 \%$ men exercise at least for the minimum time recommended by the guideline of PA (unpublished data). In this guideline, $150 \mathrm{~min}$ of moderate intensity exercise or $75 \mathrm{~min}$ of vigorous intensity exercise is considered as the minimum PA per week. ${ }^{1}$

The lower PA rate among women can be explained by gender-norm limitations that they face in their life. The limitation includes child care responsibility, security, lack of time, lack of confidence on their physical abilities, lack of knowledge about designing and maintaining a PA programme, traffic restrictions, financial inability, ${ }^{9}$ traditional views about women, weather condition, uncomfortable workout cloths and individual motivation. ${ }^{10}$

Iranian women encounter exceptional social and cultural constraints, such as disagreement with their spouse or father about going to gyms or their participation in PA. There are also some sociocultural expectations, and environmental and religious constraints, such as banning females from biking or exercising outdoor. ${ }^{11}$ As they play an important role in the nurturing and upbringing of children, being physically active is very important for women's health and could help to have healthy future generations. Undoubtedly, lack of PA among females can cause unrecoverable damages to the society as it negatively affects physical and mental health of women, a half of the population. This shows the necessity of improving PA in women. ${ }^{12}$

Although the benefits of PA are now well-established, there is not much established knowledge regarding the effectiveness of interventions designed to improve population PA. ${ }^{13}$

The fast growth of chronic diseases in developing countries has increased the awareness of correcting lifestyle inactivity and encouraged community-based interventions. Community-based interventions provide a cost-effective and reasonable way to promote health and access to PA resources for large groups of people, especially when there are limited resources within the community. ${ }^{14-16}$

Community-based interventions are multilevel approaches and use an ecological perspective. Such interventions can be implemented at any of the four ecological levels: group, organisation, community and policy. Three theories that have been used frequently in communitybased approaches are the social cognitive theory (SCT), stages of change theory and social marketing theory. ${ }^{17}$
According to Bopp and Fallon, ${ }^{14}$ community-based PA interventions involve community members and leaders from various settings and organisations (ie, at any of the four ecological levels) in the design, implementation and evaluation of a PA intervention. Owing to community members' involvement in the plan, implementation and evaluation of community-based interventions, these interventions can be more effective and sustainable than individual interventions. ${ }^{18} 19$

The majority of interventions have been delivered at the individual level to change only the personal behaviour. ${ }^{20}$ Although some individual-level and face-to-face interventions are effective as well as the gold standard for promoting $\mathrm{PA}$, transferring and delivering individual-level interventions to community-level is challenging. ${ }^{21}$ It is necessary to run the community-level interventions, which have the potential to produce long-term benefits, for a large number of people, but there is no strong evidence which type of community-based interventions are most effective. ${ }^{22}$

Although many interventions to improve PA are being carried out with women between 18 and 65 years of age, the types and effectiveness of most interventions have not been systematically examined. Recently published reviews have mostly dealt with the increase PA among both genders or only included underserved and/or minority women. Previous endeavours to summarise the evidence were mostly allocated to particular settings and individual interventions. They also did not assess the effects of interventions on women with a communitybased approach and did not assess the methodological quality of the studies.

This paper describes a systematic literature review of strategies for promoting PA among women aged 18-65 years, and conducted with community-based approaches. This review is a small part of a larger project entitled Improving PA among Women: a Mixed-method Action Research in Iran. The overarching goal of this project is to develop a community-based interventions programme for promoting PA among women in Iran.

\section{METHODS}

\section{Search strategy and inclusion criteria}

To the best knowledge of the author of this article, all documents, including articles, theses and conference abstracts, that were published between 1 January 2000 and 31 March 2013 in electronic databases, such as PubMed, Science Direct, Google scholar and Cochrane Library were searched.

The search strategy was created and run by LAF with assistance from the library and an information science expert. Keywords and combinations ( $\mathrm{MeSH}$ and text words), such as physical activity, physical inactivity, exertion, fitness and community-based intervention, community-based research and population-based intervention and community-based research, were used (table 1). 
Table 1 A sample of the search string was used in the study

\begin{tabular}{ll}
\hline Databases (hits) & Key words used \\
\hline PubMed ( $n=467)$ & (1) physical activity; (2) physical \\
Science Direct, & inactivity; (3) exertion; (4) fitness; \\
Google scholar and & (5) community-based intervention; \\
Cochrane Library & (6) community-based research; \\
$(\mathrm{n}=1643)$ & (7) population-based intervention; \\
& (8) community-based research; \\
& (9) 1 or 2 or 3 or 4 or 5 or 6 or 7 \\
& or $8 ;(10)$ randomised controlled \\
& trial; (11) controlled trial; (12) 9 \\
& and 10; (13) 9 and 11 \\
& Limit 12 and 13 to all women \\
& (18-65 years old) and English \\
& and humans \\
\hline
\end{tabular}

First, duplicate articles were removed by using End Note Software and then any remaining duplicate articles were deleted manually.

We used an iterative approach, which maximises the specifications of the search scope, to find the key literature. Additional web searches were performed after extracting relevant information, such as key words, phrases and authors, from the articles within the field of PA and community-based research (snowball search). The title and abstract of all potentially relevant articles were screened by two reviewers (LAF and OR) in order to find applicable information about PA promotion in the community-intervention section. If the abstract did not have sufficient information, the full text of the article was screened for further information. Any discrepancies between the two reviewers were resolved with discussions and consensus. If the reviewers could not reach a final conclusion, the article was investigated by the third reviewer (MA-L). The inclusion and exclusion criteria for selecting the studies were shown on the basis of PICOS in table 2.

\section{Assessment of methodological quality}

Quality assessments of studies were performed using the information available in the articles through the critical appraisal sheet. This appraisal is composed of seven scales including Delphi List, PEDro, Maastricht, Maastricht-Amsterdam List, Bizzini, vanTulder and Jadad. The appraisal was compiled in a set of 39 items by Olivo and $e t a l,{ }^{24}$ where the items were divided into five categories: patient selection, blinding, interventions, outcomes and statistics (table 3).

Each item listed in the critical appraisal sheet was specified by the score of one if it was included in the article, and specified by the score of zero if it was not included in the article or if the information provided by the authors was not sufficient to make a clear statement. In the case where a study did not consider a particular item, the item was marked as inapplicable in the critical
Table 2 The inclusion and exclusion criteria for selecting the studies on the basis of PICOS

\begin{tabular}{ll}
\hline PICOS & \\
criteria & Participants were to be 18-65 years of \\
Participants & age. \\
& The study did not involve disease-state \\
& populations (for example multiple \\
& sclerosis rehabilitation patients. \\
Interventions & Interventions must be designed to \\
& improve PA and to prevent physical \\
& inactivity, cardiovascular disease, \\
& diabetes and other side effects of \\
& sedentary life style. \\
& The study only included \\
& community-based interventions. \\
Studies must provide an assessment \\
of an intervention group through \\
comparison with a control or \\
comparison group which was \\
simultaneously derived from the same \\
or similar settings. \\
Participants were to be 18-65 years of \\
age.
\end{tabular}

appraisal sheet. The total score of each study was calculated by dividing the number of items included by the number of applicable items. The range of scores fell between zero and one. Finally, studies were graded based on the number of items that they had in the critical appraisal sheet. ${ }^{24}$ If the score was between 0 and 0.5 , it was considered a low methodological quality study, and if the score was between 0.51 and 1 , it was considered a high methodological quality study.

The critical appraisal was independently completed by the two reviewers (LAF and OR), and the results were compared. Disagreements between the two reviewers were discussed during a meeting to achieve consensus. If they could not reach an agreement, the third reviewer (MA-L) was consulted to make the final decision.

\section{Data extraction}

Standardised data extraction forms were prepared through consultation with a methodological expert. They were then verified and completed by one reviewer $(\mathrm{LAF})$, and furthermore checked by another reviewer (MA-L) for accuracy. The extracted data included the 


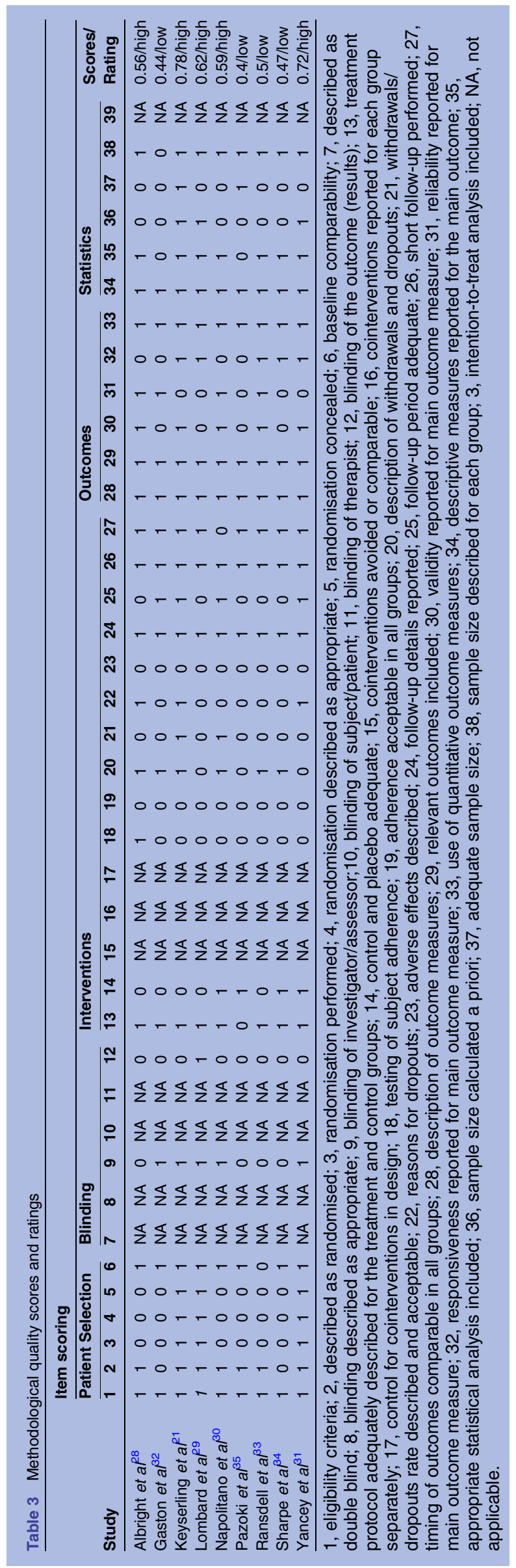

title, year, country, design of the study along with participant characteristics, randomisation procedure, intervention description, control or comparison groups, length of follow-up, measure of PA, health indicators and main results.

During the data extraction process additional details were considered: was it a theory-based intervention?; which constructs did the researchers use?; what was the number of participants at the baseline, the end of intervention and follow-up?; what was the effectiveness of the main outcome measures for assessing the level of evidence?; and, what were the details regarding the specific intervention and type of measurement tools (objective or subjective tools)?

\section{Strength of evidence and data synthesis}

Heterogeneity in the type of interventions prevented reviewers from conducting a meta-analysis of the studies; therefore, narrative synthesis was used. As previously used in best evidence syntheses, conclusions regarding the effectiveness of programmes on PA outcome measures were drawn using a rating system referencing the levels of evidence on the basis of study design and methodological quality. ${ }^{25-27}$ Five levels of evidence were defined: (1) strong evidence: at least 2 RCTs of high quality with 'consistent' (significant) results; (2) moderate evidence: 1 RCT of high quality and at least 1 RCT of low quality, or 1 RCT of high quality and at least 1 controlled trial of high quality (for both situations, consistent results were required); (3) limited evidence: 1 RCT of high quality and at least 1 controlled trial of low quality or more than 1 RCT of low quality or more than 1 controlled trial of high quality (for all situations, consistent results were required); (4) inconclusive evidence: only 1 study or multiple-controlled trials of low quality or contradictory results; and (5) no evidence: more than 1 study with no significant or relevant results to a specific intervention. When the results of the studies were considered with regard to statistical significance, the $p$ value was less than 0.05 . If at least $75 \%$ of each of the relevant studies were reported to have significant results in the same direction then we considered the overall results to be consistent'. In a stratified analysis we assessed and reported levels of evidence for studies according to type of intervention.

\section{RESULTS}

Overall, the initial search identified 2110 publications. After deduplication, 1218 relevant articles remained. At first the screening of titles led to 315 potentially relevant articles. The abstract content of all 315 studies were then screened and finally 53 articles remained, which had inclusion and exclusion criteria of systematic review on the basis of titles and abstracts assessment. The full text of the 25 studies were included for details assessment, resulting in 16 articles being excluded (figure 1). The reasons for exclusion were irrelevant outcomes for 
this review $(\mathrm{n}=5)$, PA interventions without evaluation $(\mathrm{n}=4)$, non-community-based intervention $(\mathrm{n}=2)$, involvement of disease-state populations and participants who were more than 65 years of age in the study $(n=3)$, publication of two similar articles in different journals $(n=1)$ and the use convenience sampling $(n=1)$.

Nine articles were selected from this literature review. Table 4 provides the characteristics (ie, population, general intervention, outcome measure, measurement times and results) of all studies included in the review.

\section{Methodological quality}

Table 3 shows the methodological quality of the included studies. Agreement was $92.6 \%$ on the 325 items scored through the quality assessment. Full consensus on all items was reached after discussion between the two reviewers. Five of the 9 articles were considered high quality. ${ }^{21} 28-3031$ There was not sufficient information about random allocation used in most studies as only 3 of these $(33 \%)$ described random allocation 212931 and only $3(33 \%)$ provided sufficient information about allocation concealment at the time of outcome assessment. ${ }^{21} 2931$ There were blinding issues due to nature of PA interventions as it was not possible to blind participants to the types of intervention. However, some studies used blinding of investigator/assessor and statistician to increase study accuracy. Five studies (55\%) applied blinding of the investigator ${ }^{21}{ }^{29-32}$ and 1 study (11\%) solely used blinding of statistician. ${ }^{29}$ Most studies had similar periods which passed before conducting the outcome assessment. Only 4 studies (44\%) had a follow-up of 3 months or longer. ${ }^{21}$ 30-32

\section{Study characteristics}

Seven of 9 studies were carried out in the USA, 2128 30-34 1 in Australia, ${ }^{29}$ and 1 in Iran. ${ }^{35}$

The intervention studies were categorised as: physical activity only, nutritional and physical activity interventions. There were 5 of nine articles where programmes were designed to modify $\mathrm{PA}^{283033-35}$ and the remainder

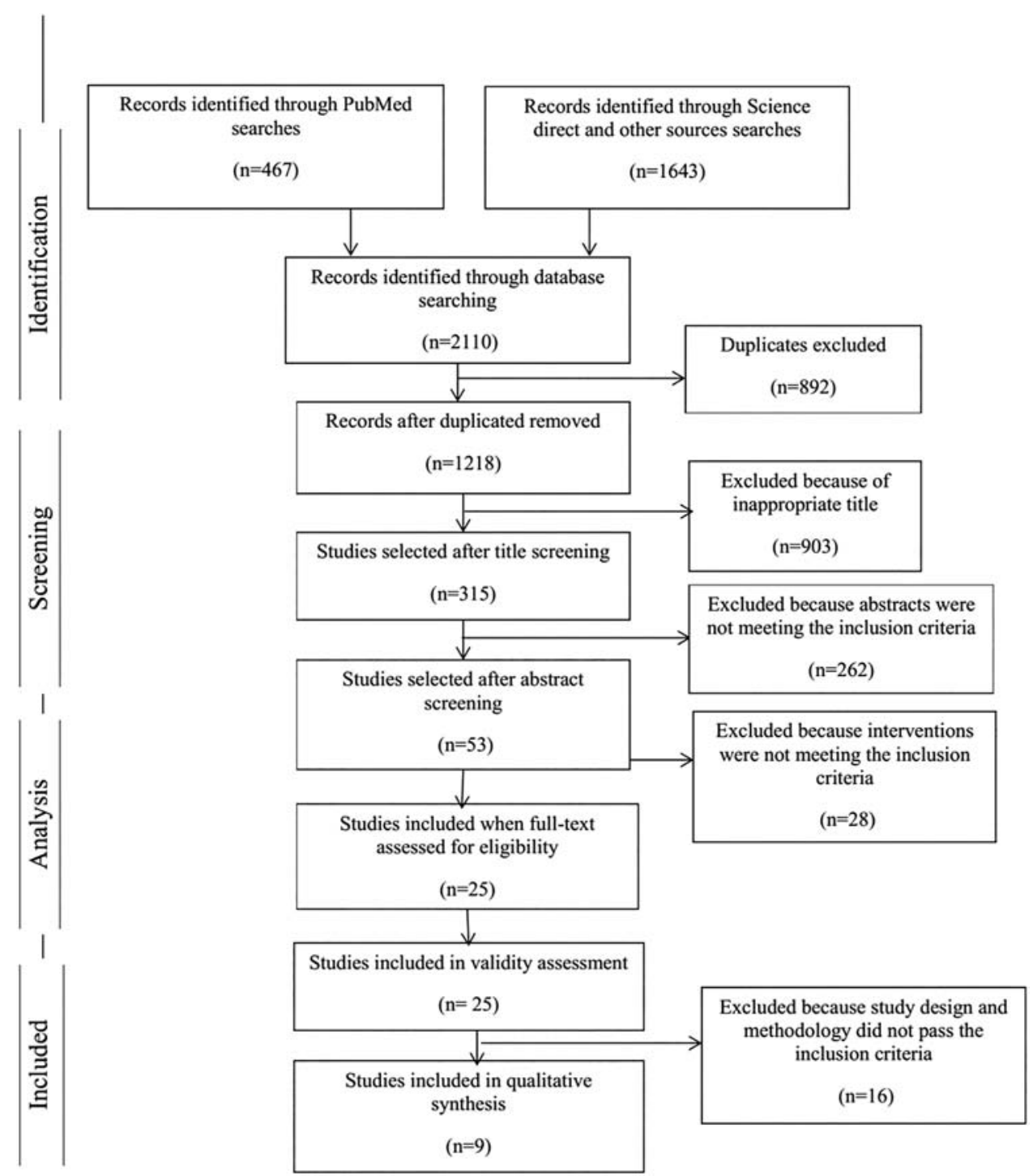

Figure 1 Flow diagram used for the identification, screening, eligibility and inclusion of studies. ${ }^{23}$ 
were designed as both PA and nutritional interventions. $^{21} 29$ 31-32

The most common duration for interventions was 12 months. $^{21} 2834$ Other interventions lasted 8 weeks, ${ }^{31} 35$ 10 weeks $^{32}$ or 4 months. ${ }^{29}$

All of the studies were designed on the basis of a multicomponent approach. All studies evaluated social science theory-based interventions; seven of nine studies used applied social cognitive theories, ${ }^{21}$ 28-30 $3233 \quad 35$ while 1 used both SCT and social marketing theory (SMT), ${ }^{34}$ and another used the social ecological model. ${ }^{31}$ The most common constructs of SCT were used, including social support, goal setting, overcoming potential barriers and self-monitoring. Some studies have emphasised specific constructs or applied particular interventions that did not exist in other studies. For example, Albright et $a l^{28}$ used verbal encouragement and written reinforcement to achieve short-term and long-term PA goals. Gaston $e t a l^{2}$ and Pazoki et $a l^{35}$ used cultural facilitators and expert consultants for teaching behavioural strategies and skills to help the women implement an individualised health plan. Keyserling et $a l^{21}$ gave contact information to participants for local healthy PA resources. Lombard et $a l^{29}$ offered problem-solving training for overcoming the barriers of PA. Ransdell et $a l^{33}$ used a daughter and mother exercise strategy to produce social support and motivation to increase PA. Sharpe et al used media messages for promotion of PA. ${ }^{34}$ Yancey $e t a l^{31}$ applied an economic incentive of a free 1-year gym membership for all participants.

Measurement of PA was mostly focused on self-report questionnaires or recall instruments (using different types of PA questionnaire). Four of nine articles used both self-report questionnaires or recall instruments and pedometers for measurement of PA. ${ }^{21} 28-2934$

\section{Evidence of effect on physical activity}

Seven studies reported a positive intervention effect $(77.7 \%)$, and in 4 of these studies statistical significance was achieved $(44.45 \%)$. Significant results ranged from an increase of 2.07 days per week in doing aerobic exercise to a $10.4 \%$ increase in participation in regular PA (at least $30 \mathrm{~min}$ of moderate intensity PA for at least 5 days a week, or at least $20 \mathrm{~min}$ of vigorous PA for at least 3 days a week).

Seven studies evaluated social cognitive theory-based interventions, including 2 high-quality randomised controlled trials, ${ }^{2129} 2$ high quality controlled trials ${ }^{28} 30$ and 3 low quality controlled trial. ${ }^{32} 3335$ Two of these studies were high quality and randomised controlled trials, ${ }^{21} 29$ but had no statistically significant intervention effect; therefore, there was no evidence on the basis of effectiveness for social cognitive theory-based interventions.

With regard to other social science theory-based interventions, there was only 1 low quality controlled trial intervention accomplished on the basis of a mix of SCT and SMT, and 1 high-quality randomised controlled trial which used the social ecological model. ${ }^{31} 34$ These two articles illustrated the inconclusive evidence of intervention effectiveness.

\section{DISCUSSION}

\section{Summary of evidence}

The purpose of this systematic literature review was to assess the effectiveness of community-based PA interventions for women. Many studies were found in the literature, but a very small number of studies were community-based interventions performed among women or met the inclusion criteria of this study. Consequently, this problem brought about a small number of studies being included in the review. Most of these studies modified PA and were multicomponent interventions. However, reviewers attempted to categorise the studies in a meaningful and logical model, but were unable to recognise any consistent evidence to support the effectiveness of community-based interventions to enhance PA level. Heterogeneity existed between the types of interventions, intensity of activities, study designs, the duration of follow-ups and assessment tools. Reviewers found that social cognitive theory-based interventions had no evidence of an effect of interventions on PA and the evidence of an effect for other social science theory-based interventions was inconclusive. Most of these studies were not random and did not have any statistical significance. More high quality and randomised studies are required to strengthen and confirm these results. In overall, due to specific characteristics of interventions, reviewers could not determine which type of interventions, intensity, frequency or type of PA were successful in promoting PA among women.

\section{Implementation of interventions}

Results showed that most of the articles were limited or had inconclusive evidence of an interventions' effect. There were many factors which contributed to the restricted effectiveness of interventions: small sample size, small power to detect differences between groups, baseline differences between groups, the intensity levels of interventions, lack of wait-list control group by comparing the intervention group results with another intervention type or minimal intervention, and adherence. Several studies which were included had these problems. For example, all of the papers described did not have acceptable adherence and most of them did not have a control group. All studies had a sociological basis; however, even those that used same theories had different constructs.

\section{Limitations and recommendation for future studies}

There are a number of limitations to this study. First, reviewers limited the search to English language articles and did not include other language interventions, such as German or Italian. Second, the search strategy covered resources published between 2000 and 2013 as 


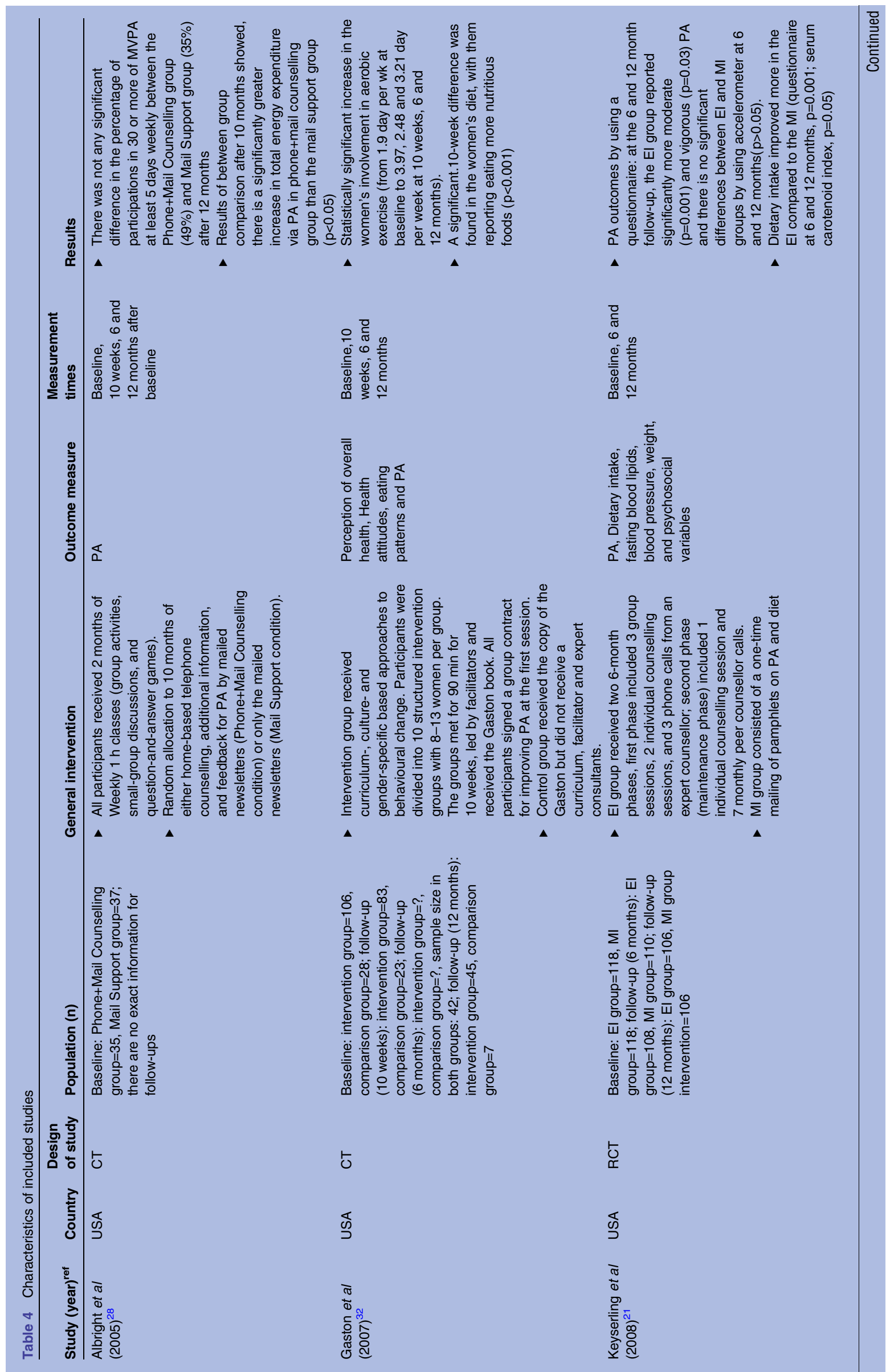




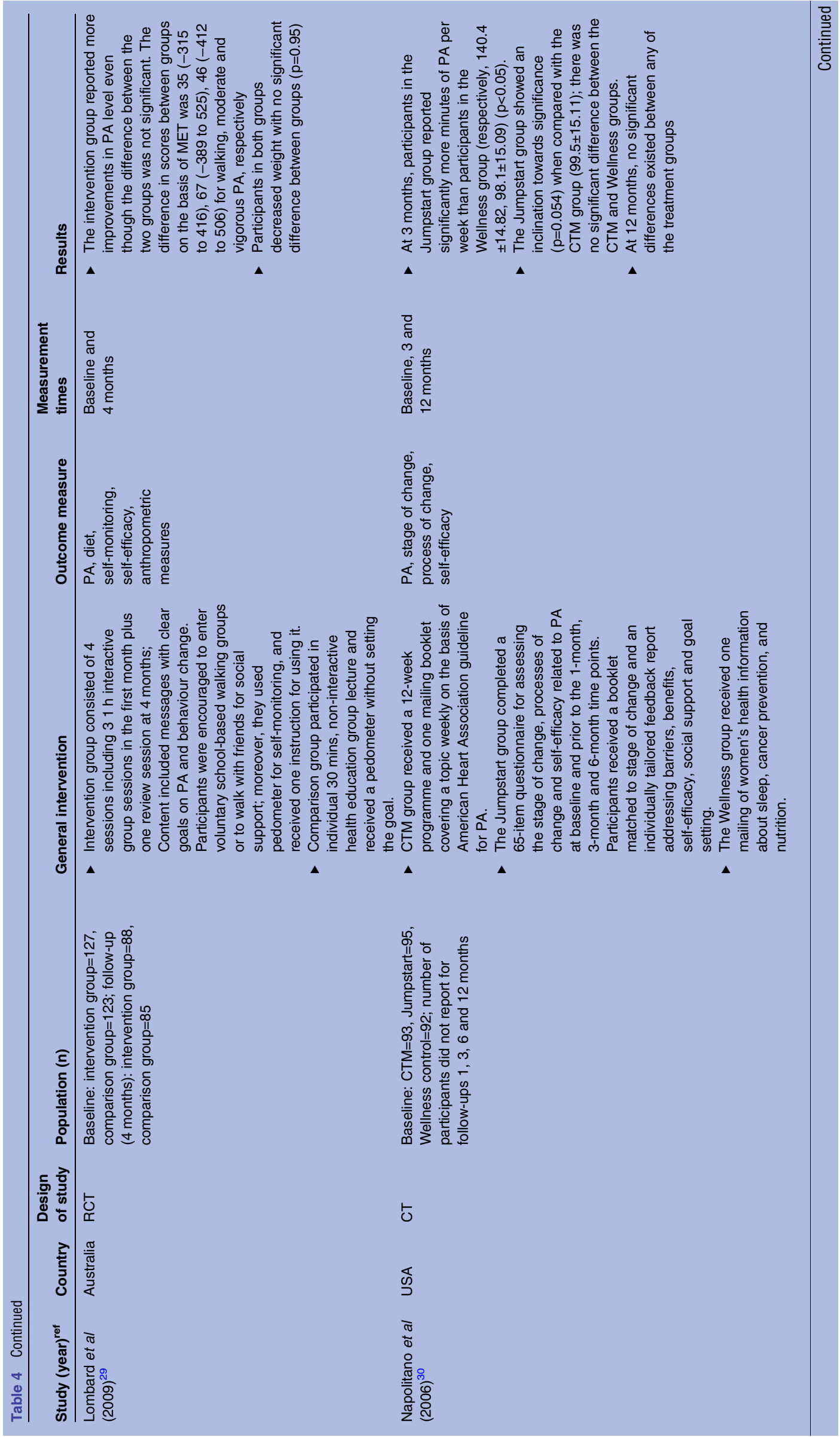




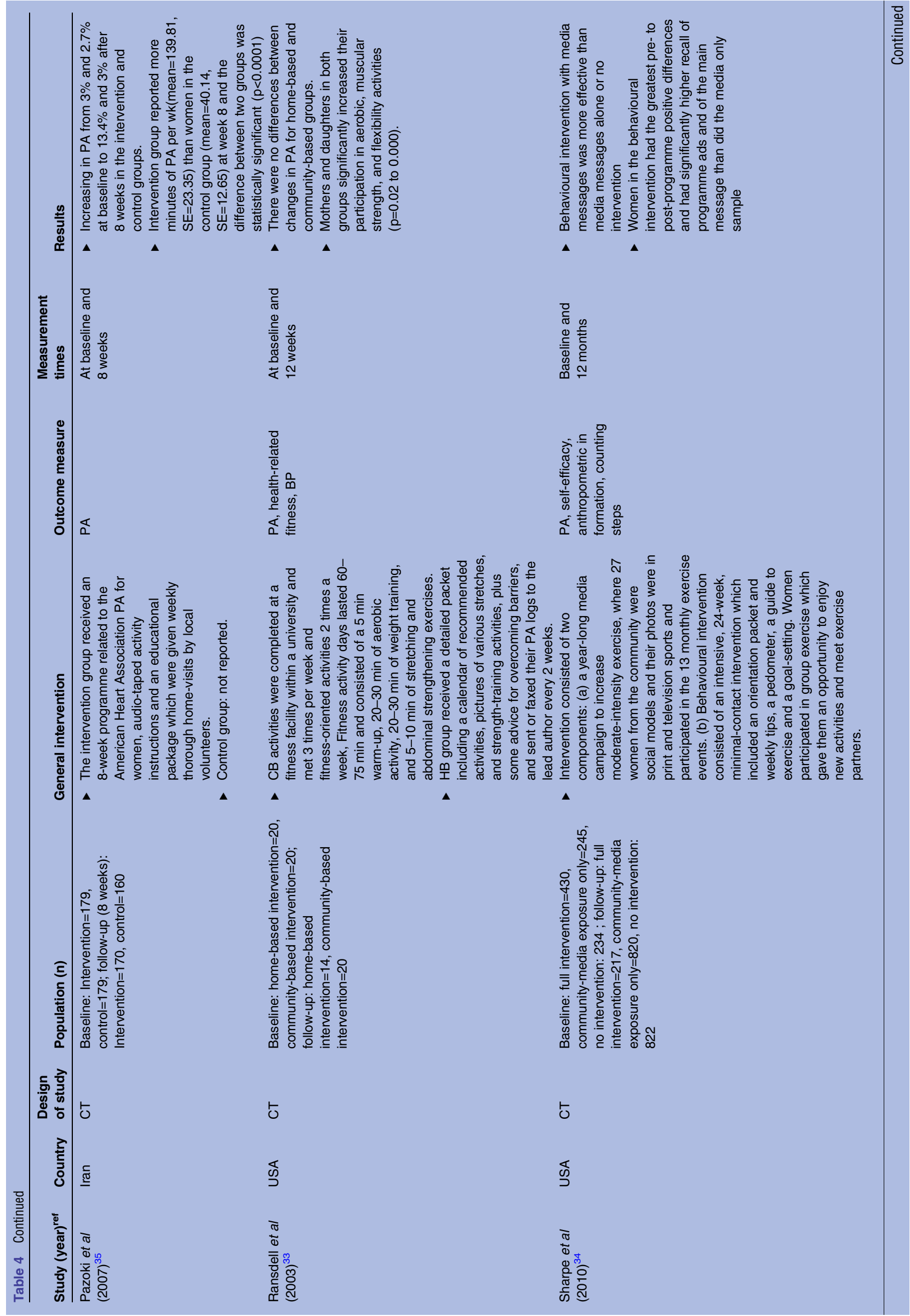




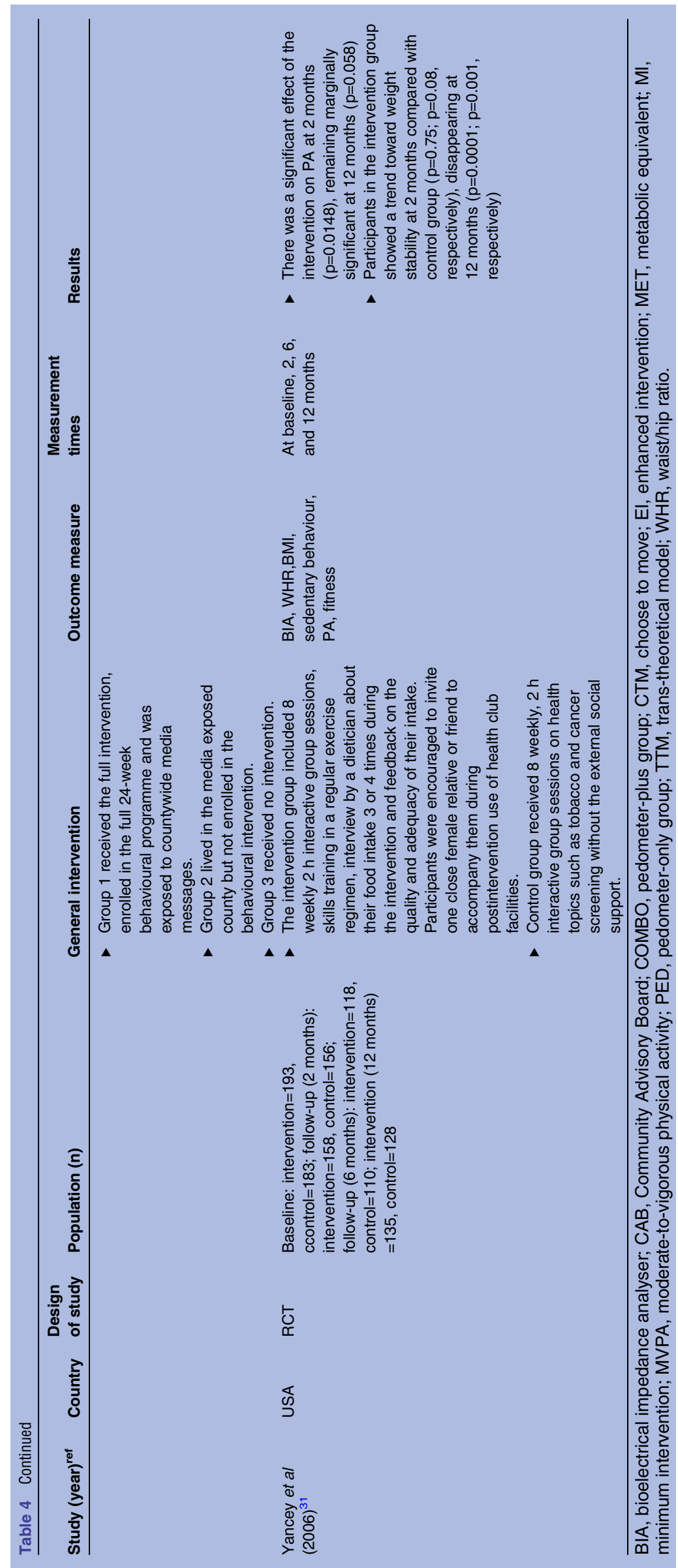


the process of conducting the systematic review and reviewing the article was long.

Third, due to the small number of included papers and the lack of statistically significant differences, the results of this review are difficult to interpret. Fourth, methodological limitations across studies included the short time of intervention or follow-up, insufficient adjustment for potential confounders, lack of randomisation procedure and blinding at outcome assessment. Fifth, there was a lack of precision in the measurement of PA outcomes in some studies. Sixth, a conclusive meta-analysis cannot be achieved with these studies because of the heterogeneous nature of these studies and explanations cannot be made concerning the effect size of the interventions. Seventh, reviewers could not distinct biased publications that only reported positive findings in community-based interventions for PA improvement as these publications were some of the available resources. Reviewers also faced the challenge that measures of PA differed markedly and were reported both as indirect and direct measures. Though reviewers had planned a priori to conduct subgroup analysis of direct (eg, accelerometer or pedometer) versus indirect (eg, self-report) measures of PA, this was not possible because of the heterogeneity of measurement tools and interventions.

To have a fair assessment, future studies on PA measurement should have similar approaches and tools. There is a need for more rigorous research designs, including higher quality randomised controlled trials in this age group and culture-based multicomponent and community-based intervention programmes that consider either individual or environmental factors for changing PA levels

One of the goals of the community intervention is to design programmes that include the majority of the population, but it seems including personal desires and interests into the design of PA programmes could provide better results. One intervention approach may not fit all, therefore, different approaches should be offered: some people may prefer the private feedback from a device such as pedometer; others may respond to interventions delivered through the internet, others may benefit from the social support in doing a PA group, whereas others may increase PA in response to telephone counselling or facilitator counselling.

In community-based interventions, the number of participants that contribute in all levels of measurement, design, application and assessment increase the chance of success for an intervention programme. At the same time, the efficacy and reliability of an intervention programme is more important than the number of people that an intervention could involve.

\section{CONCLUSION}

To our extensive search, this is the first published systematic review aimed at community-based PA intervention studies for 18-65 years-old women. This review found low-quality to high-quality evidence of how to improve $\mathrm{PA}$, although due to the inadequate supply of information reviewers could not determine which specific type, intensity, frequency or amount of intervention could significantly improve PA, or which intervention is more effective and sustainable. In addition, more studies are needed to address these gaps in knowledge for PA improvement among women. Based on the published evidence to date, it is necessary to conduct a multilevel approach for promoting PA. Reviewers have recognised the necessity of collaborations among community members, policymakers, as well as governmental and non-governmental organisations in developing more effective PA interventions for women.

\section{Author affiliations}

${ }^{1}$ Department of Midwifery, School of Nursing and Midwifery, Tehran University of Medical Sciences, Tehran, Iran

${ }^{2}$ DG Int'I Relations Department, MOHME, IR Iran Head- Oncopathology Research Centre, IUMS, Tehran, Iran

${ }^{3}$ Nursing Department, Faculty of Medical Sciences, Tarbiat Modares University, Tehran, Iran

${ }^{4}$ Department of Pediatric Nursing, Nursing and Midwifery Faculty, Iran University of Medical Sciences, Tehran, Iran

${ }^{5}$ Centre for educational Research in Medical Sciences (CERMS) Iran University of Medical Sciences, Tehran, Iran

${ }^{6}$ The Research Centre for Modelling in Health, Institute for Future Studies in Health, Kerman University of Medical Sciences, Kerman, Iran

${ }^{7}$ Faculty Member of Nursing and Midwifery Care Research Centre, Nursing and Midwifery School, Tehran University of Medical Sciences, Tehran, Iran

Acknowledgements The authors would like to thank Dr Susan Armijo-Olivo, Adjunct Professor at the Faculty of Rehabilitation Medicine, University of Alberta, Canada, for her constructive help, Dr Owais Reza (OR), PhD candidate in clinical epidemiology at Tehran University of Medical Sciences for his help and consultation throughout some steps of the study, Kayla J Power for her valuable and kind assistance in finding the full text of articles and English language editing of the article.

Contributors MA-L and LAF conceived and designed the review. MAL, LAF and $\mathrm{OR}$ extracted and analysed the data. EM, SP and ZT wrote the paper. AAH revised the paper.

Funding This work is a part of the author's PhD thesis in Tehran University of Medical Sciences. This study was funded and supported by Tehran University of Medical Sciences (grant no. 93-02-28-26113).

Competing interests None.

Provenance and peer review Not commissioned; externally peer reviewed.

Data sharing statement No additional data available.

Open Access This is an Open Access article distributed in accordance with the terms of the Creative Commons Attribution (CC BY 4.0) license, which permits others to distribute, remix, adapt and build upon this work, for commercial use, provided the original work is properly cited. See: http:// creativecommons.org/licenses/by/4.0/

\section{REFERENCES}

1. Department of Health and Human Services. Physical Activity Guidelines Advisory Committee report 2008. Washington, DC: Department of Health and Human Services, 2008.

2. Nikolic IA, Stanciole AE, Zaydman M. Chronic emergency: why NCDs matter. Health, Nutrition, and Population Discussion Paper; 2011.

3. World Health Organization. Global recommendations on physical activity for health. 2010. http://whqlibdoc.who.int/publications/2010/ 9789241599979_eng.pdf 
4. World Health Organization. Global strategy on diet, physical activity, and health 2006. Geneva: World Health Organization, 2009.

5. Hoehner CM, Soares J, Parra Perez D, et al. Physical activity interventions in Latin America: a systematic review. Am J Prev Med 2008;34:224-33.

6. Friedenreich $\mathrm{CM}$. Physical activity and cancer prevention from observational to intervention research. Cancer Epidemiol Biomarkers Prev 2001;10:287-301.

7. Macera CA, Ham SA, Yore MM, et al. Prevalence of physical activity in the United States: behavioral risk factor surveillance system, 2001. Prev Chronic Dis 2005;2:A17.

8. Dumith SC, Hallal PC, Reis RS, et al. Worldwide prevalence of physical inactivity and its association with human development index in 76 countries. Prev Med 2011;53:24-8.

9. Wilcox S Bopp M, Oberrecht L, et al. Psychosocial and perceived environmental correlates of physical activity in rural and older african american and white women. J Gerontol B Psychol Sci Soc Sci 2003;58:P329-37.

10. Berger G, Peerson A. Giving young Emirati womena voice: participatory action research on physical activity. Health Place 2009;15:117-24.

11. Baheiraei A, Mirghafourvand M, Mohammad-Alizadeh Charandabi S, et al. Facilitators and inhibitors of health-promoting behaviors: the experience of Iranian women of reproductive age. Int J Prev Med 2013;4:929-39.

12. Centres for Disease Control and Prevention. Behavioral risk factor surveillance system data. Atlanta, GA: U.S. Department of Health and Human Services, 2007.

13. Hillsdon M, Foster C, Thorogood M. Interventions for promoting physical activity. Cochrane Database Syst Rev 2005;(1):CD003180.

14. Bopp M, Fallon E. Community-based interventions to promote increased physical activity: a primer. Appl Health Econ Health Policy 2008;6:173-87.

15. Garrett S, Elley CR, Rose SB, et al. Are physical activity interventions in primary care and the community cost-effective? A systematic review of the evidence. Br J Gen Pract 2011;61:e125.

16. Harding $\mathrm{AH}$, Griffin SJ, Wareham NJ. Population impact of strategies for identifying groups at high risk of type 2 diabetes. Prev Med 2006;42:364-8.

17. Guttmacher S, Kelly PJ, Ruiz-Janecko Y. Community-based health interventions. San Francisco: Jossey-Bass, 2010.

18. Economos CD, Irish-Hauser S. Community interventions: a brief overview and their application to the obesity epidemic. J Law Med Ethics 2007;35:131-7.

19. Israel BA, Schulz AJ, Parker EA, et al. Review of community-based research: assessing partnership approaches to improve public health. Annu Rev Public Health 1998;19:173-202.

20. Solomon E, Rees T, Ukoumunne OC, et al. The Devon Active Villages Evaluation (DAVE) trial: Study protocol of a stepped wedge cluster randomised trial of a community-level physical activity intervention in rural southwest England. BMC Public Health 2012;12:581.

21. Keyserling TC, Samuel Hodge CD, Jilcott SB, et al. Randomized trial of a clinic-based, community-supported, lifestyle intervention to improve physical activity and diet: The North Carolina enhanced WISEWOMAN project. Prev Med 2008;46:499-510.

22. Baker PR, Francis DP, Soares J, et al. Community wide interventions for increasing physical activity. Cochrane Database Syst Rev 2011;(4):CD008366.

23. Moher D, Liberati A, Tetzlaff $\mathrm{J}$, et al. Preferred reporting items for systematic reviews and meta-analyses: the PRISMA statement. Int J Surg 2010;8:336-41.

24. Olivo SA, Macedo LG, Gadotti IC, et al. Scales to assess the quality of randomized controlled trials: a systematic review. Phys Ther 2008;88:156-75.

25. Proper KI, Staal BJ, Hildebrandt VH, et al. Effectiveness of physical activity programs at worksites with respect to work-related outcomes. Scand J Work Environ Health 2002;28:75-84.

26. Engbers LH, van Poppel MN, Chin A et al. Worksite health promotion programs with environmental changes: a systematic review. Am J Prev Med 2005;29:61-70.

27. van Sluijs EM, van Poppel MN, van Mechelen W. Stage-based lifestyle interventions in primary care: are they effective? Am J Prev Med 2004;26:330-43.

28. Albright CL, Pruitt L, Castro C, et al. Modifying physical activity in a multiethnic sample of low-income women: one-year results from the IMPACT (Increasing Motivation for Physical ACTivity) project. Ann Behav Med 2005;30:191-200.

29. Lombard CB, Deeks AA, Ball K, et al. Weight, physical activity and dietary behavior change in young mothers: short term results of the HeLP-her cluster randomized controlled trial. Nutr $J$ 2009;8:17

30. Napolitano MA, Whiteley JA, Papandonatos G, et al. Outcomes from the women's wellness project: a community-focused physical activity trial for women. Prev Med 2006;43:447-53.

31. Yancey AK, McCarthy WJ, Harrison GG, et al. Challenges in improving fitness: results of a community-based, randomized controlled lifestyle change intervention. J Womens Health (Larchmt) 2006;15:412-29.

32. Gaston MH, Porter GK, Thomas VG. Prime Time Sister Circles: evaluating a gender-specific, culturally relevant health intervention to decrease major risk factors in mid-life African-American women. J Natl Med Assoc 2007;99:428-38.

33. Ransdell LB, Taylor A, Oakland D, et al. Daughters and mothers exercising together: effects of home- and community-based programs. Med Sci Sports Exerc 2003;35:286-96.

34. Sharpe PA, Burroughs EL, Granner ML, et al. Impact of a community-based prevention marketing intervention to promote physical activity among middle-aged women. Health Educ Behav 2010;37:403-23.

35. Pazoki R, Nabipour I, Seyednezami N, et al. Effects of a community-based healthy heart program on increasing healthy women's physical activity: a randomized controlled trial guided by Community-based Participatory Research (CBPR). BMC Public Health 2007;7:216. 